\title{
Synthesis and characterisation of hot extruded aluminium-based MMC developed by powder metallurgy route
}

\author{
Sambit Kumar Mohapatra and Kalipada Maity ${ }^{*}$
}

\begin{abstract}
Background: Improvement of the mechanical and tribological properties due to extrusion can be attributed to the improved density and excellent bond strength due to high compressive stress.

Methods: To avoid the product defects mathematically contoured cosine profiled die was used for the thermomechanical treatment. Improvement of mechanical characterization like density, hardness, compression test and three-point-bend test was inquired. Two body dry sliding wear behaviour of the prepared AMCs before and after extrusion were investigated by using a pin-on-disc wear testing method by varying the variable parameters like load $(\mathrm{N})$, track diameter ( $\mathrm{mm}$ ) and RPM of the counter disc.

Results: The effect of hot extrusion on mechanical and tribological characteristics of aluminium matrix composites (AMCs) developed by powder metallurgy route followed by double axial cold compaction and controlled atmospheric sintering was studied.

Conclusion: Shearing of the fine distributed graphite particles at the tribosurface acts as a solid lubricant and decreases wear rate. At higher loading and sliding velocity condition a mixed type of wear mechanism was observed.
\end{abstract}

Keywords: AMCs, Extrusion, Density, Wear, P/M

\section{Background}

Aluminium metal matrix composites are the most versatile replacement of other alloys in the sector of automotive, aerospace, defence and sports because of its high strength to weight ratio, ease and prevalence of processing techniques, excellent thermal and electrical conductivity and the ability to sustain in uncertain thermal and mechanical loading environment. This emerging area has influenced the researchers to tailor the mechanical, thermal and tribological properties of the composite using different types of reinforcements with various percentages with types of manufacturing process (Yigezu et al. 2013). The endless process of pursuance of mankind needs the material to behave substantially in critical environments.

\footnotetext{
* Correspondence: kpmaity@gmail.com

Department of Mechanical Engineering, National Institute of Technology, Rourkela, Odisha 769008, India
}

Over the last few decades, there has been considerable attention to the evolution of Al-based MMCs developed by powder metallurgy $(\mathrm{P} / \mathrm{M})$ route of manufacturing. The main advantage of this kind of manufacturing process is the good distribution of reinforcing particles, low processing temperature and the ability to produce near net shape products with intricate designs (Min et al. 2005; Torralba et al. 2003). A number of studies have been conducted to study the effects of reinforcement of very hard metals as well as ceramics in different grades of aluminium series of powder matrix (El-Kady and Fathy 2014; Jabbari Taleghani et al. 2014; Abdollahi et al. 2014). Layers of oxide formation take place in the $\mathrm{P} / \mathrm{M}$ specimen during sintering, as aluminium is highly prone to oxide formation. During thermo-mechanical treatments, the covered oxide layer breaks due to highly induced shear stress, leading to a strongly bonded microstructure and improved mechanical properties which eliminate the main drawback of AMCs (Schatt et al. 1997). The use of traditional shear- 
Table 1 Detailed composition

\begin{tabular}{ll}
\hline Specimen & Composition \\
\hline Type 1 & $\mathrm{Al}(92.33)+\mathrm{Mg}(4.26)+\mathrm{Gr}(0.85)+1.70 \mathrm{Zn}$ \\
Type 2 & $\mathrm{Al}(92.33)+\mathrm{Mg}(4.26)+\mathrm{Gr}(0.85)+1.70 \mathrm{Ti}$ \\
\hline
\end{tabular}

faced die in extrusion causes product defects owing to the existence of higher velocity relative difference at die exit (Zhang et al. 2012a; 2012b). The use of mathematically contoured die (preferably zero, die entry and exit angle) for the MMC extrusion is highly recommendable.

Ravindran et al. (2012; 2013) investigated the effect of graphite addition, applied load, relative velocity and sliding distance on the wear behaviour of aluminium-based P/M composite. Fine graphite reinforcement acts as a solid lubricant and prevents metal to metal contact so it improves wear resistance compromising with hardness and flexural strength or fracture toughness (Baradeswaran and Perumal 2014; Suresha and Sridhara 2010). The addition of zinc with aluminium improves hot extrudability but decreases the high-temperature performances. Considering these factors, the weight percentage of reinforcements kept less in this work. Improved amount of $\mathrm{TiC}$ causes a marginal increase in wear rate, whereas applied load and wear rate varies linearly (Gopalakrishnan and Murugan 2012). Anilkumar et al. (2011) investigated the mechanical properties of the fly ash reinforced aluminium alloy. Improvement of mechanical properties can be achieved by adding more amount of reinforcement by compromising with ductility.

The present investigation focuses on both mechanical and tribological properties of extruded AMC synthesised by powder metallurgy route. Two types of metal reinforcements like $\mathrm{Zn}$ and $\mathrm{Ti}$ in $\mathrm{Al}+\mathrm{Mg}+\mathrm{Gr}$ matrix has been added for the comparative study. The properties of AMCs before and after thermo-mechanical treatment (extrusion) through a mathematically contoured cosine die were inquired.

\section{Methods}

\section{Sample preparation}

Aluminium, magnesium and graphite in weight percentages of 92.33, 4.26 and 0.85 , respectively, were blended for the matrix composition. Two reinforcements, $\mathrm{Zn}$ and $\mathrm{Ti}$ were added in the mixture for preparing two types of sample. The compositional details of two specimens were tabulated in Table 1. The physical characterisation of the powders like size, shape and flowability was studied. Particle size and shape was analysed by SEM images.

The mixture was allowed for uniform blending in a centrifugal blender. The weight ratio of stainless steel ball to powder was maintained 10:1. At an RPM of 200 for $10 \mathrm{~h}$, the mixture was allowed for blending. The flow property of the blended powders was checked by measuring apparent density and tap density of the blended compositions.

The powder was subjected to dual axial compression for the preparation of green pellets. During compaction, the powder inside the container remains in floating condition in between both of the punches. The powder was subjected to a pressure of $275 \mathrm{MPa}$ with a very slow rate of rise and with a dwell period of $10 \mathrm{~min}$. Green density of the prepared 10-mm-diameter pellets was measured. Green pellets were subjected to sintering in a controlled atmospheric tubular furnace in an argon atmosphere. The ramp rate of $5{ }^{\circ} \mathrm{C} / \mathrm{min}$ was set for all the temperature rises. Dwell period of $20 \mathrm{~min}$ at $110{ }^{\circ} \mathrm{C}$ to remove water vapour, $30 \mathrm{~min}$ at $450{ }^{\circ} \mathrm{C}$ to remove lubricant (zinc stearate) and $90 \mathrm{~min}$ at $590{ }^{\circ} \mathrm{C}$ to form the metallic bond was set for the process.

\section{Secondary processing}

The 10-mm-diameter pellet prepared by cold compaction followed by sintering was subjected to thermomechanical treatment (hot extrusion) as a secondary processing. The experiment was conducted for 50\% reduction of the cross-sectional area of the pellet at an

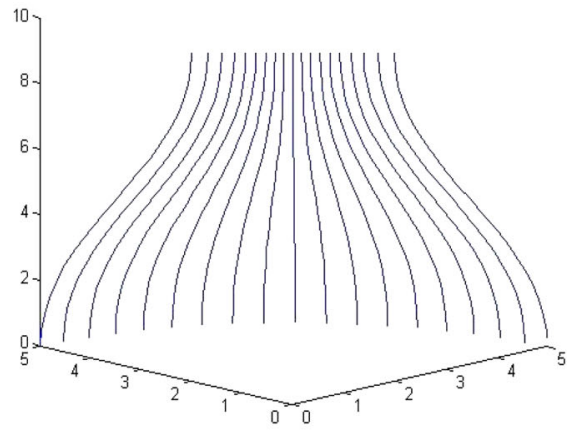

(a)

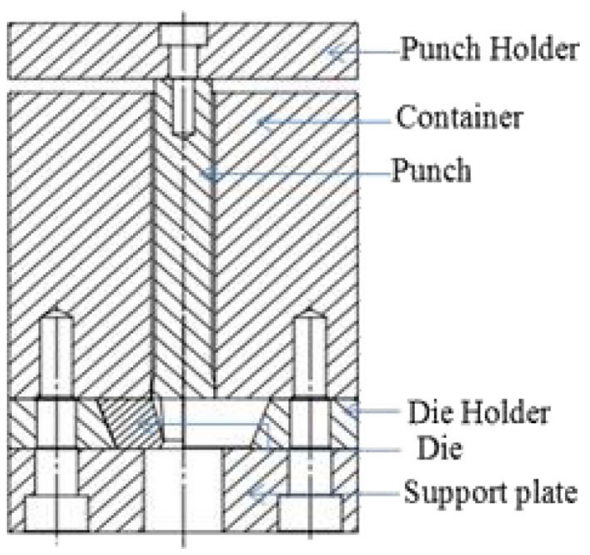

(b)

Fig. 1 a Profile coordinates of the cosine die profile in one quadrant. b 2D drafting of the tooling setup 


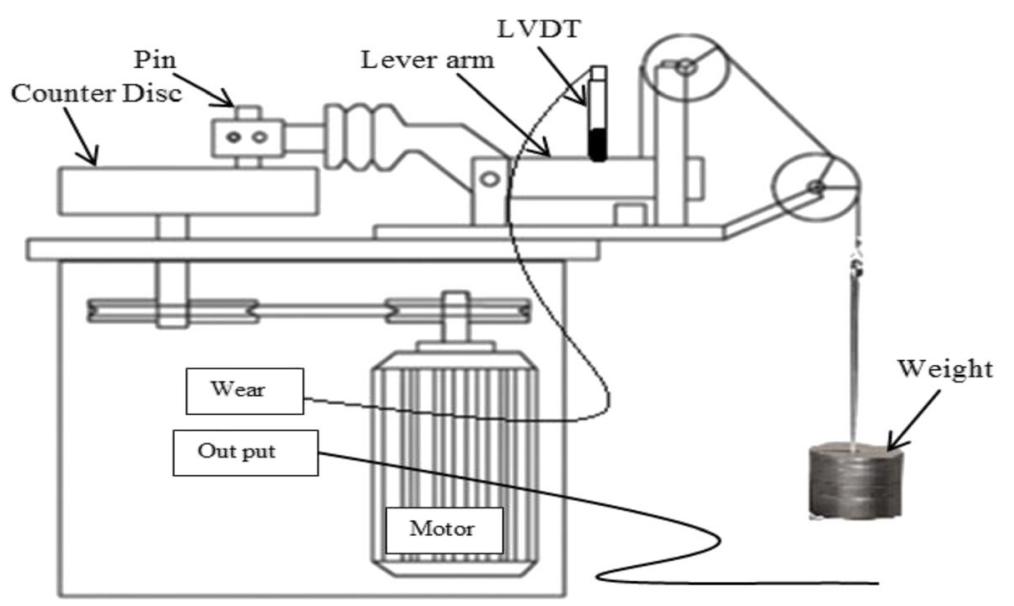

Fig. 2 Schematic layout of pin-on-disc wear testing apparatus

operating temperature of $400-450{ }^{\circ} \mathrm{C}$ with a ram rate of $3 \mathrm{~mm} / \mathrm{min}$. Extrusion through shear-faced die causes severe surface defects like surface crack and tearing in the extruded product due to velocity relative difference at the die exit. The defects are more prone in the case of extrusion of MMCs synthesised by powder metallurgy route (Prasad et al. 2001). Hence, a specially designed mathematically contoured cosine die from round to square bar extrusion was used for extrusion to avoid severe velocity relative difference. The coordinates of developed profile in one quadrant and the $2 \mathrm{D}$ drafting of the extrusion tooling setup are shown in Fig. 1.

\section{Mechanical and tribological characteristics}

Theoretical density, tap density, green density, sintered density and extruded density were studied by following the standard procedures. Theoretical density was measured by following the relation mentioned in Eq. 1.

$$
\rho_{\text {Theoretical }}=\sum\left(\rho_{\mathrm{i}} \times m_{\mathrm{i}}\right)
$$

$\rho_{\mathrm{i}}$ is the density of individual element and $m_{\mathrm{i}}$ is the mass fraction of the individual element.

The density of the solid-sintered as well as extruded pellets were measured by following Archimedes' principle. The relation for calculating the density is presented in Eq. 2.

$$
\rho_{\text {sample }}=\frac{W_{\mathrm{A}} \times \rho_{\text {fluid }}}{W_{\mathrm{A}}-W_{\text {fluid }}}
$$

where $W_{\mathrm{A}}$ is the mass of the sample taken at atmospheric air, $W_{\text {fluid }}$ is the mass of the considered fluid and $\rho_{\text {fluid }}$ is the density of the considered fluid.

Vickers micro-hardness of the sintered MMC composite was determined by dividing the applied load to the impressed area. $50 \mathrm{~g}$ of load were applied through a diamond pyramid having the face angle of $136^{\circ}$ for a dwell period of $15 \mathrm{~s}$ to avoid spring back effects.

Three-point bend test has been performed to check the transverse rupture strength (TRS) of the sintered solid cylindrical specimen. The test was executed in UTM (universal testing machine, Instron -5979). A span of $30 \mathrm{~mm}$ with a compression rate of $2 \mathrm{~mm} / \mathrm{min}$ at atmospheric temperature was maintained at the time of operation.

\section{Wear testing}

Dry sliding wear characteristics of the prepared aluminium MMC was studied by pin-on-disc wear testing apparatus. The pins with dimension $\varnothing 10 \times 25 \mathrm{~mm}$ were prepared with flat contact surface with smooth corners and kept stationary in the sample holder perpendicular to the counter disc. The rotating EN-31 disc of $60 \mathrm{HRC}$ and average surface roughness value of $2 \mu \mathrm{m}$ ( $\mathrm{Ra}$ ) was acted as the counter body. The counter disc and pin surface were cleaned with acetone before the experimentation. A normal load was applied on the MMC pellet through the specimen holder by a lever attachment. The line diagram of wear testing mechanism is shown in Fig. 2. The variable parameters like track diameter, normal load and RPM of the disc are well facilitated by the machine set-up, and all are needed to be fixed manually

Table 2 Variable parameters selected for experimentation

\begin{tabular}{llll}
\hline Variable parameters & Level 1 & Level 2 & Level 3 \\
\hline Wear track dia $(D),(\mathrm{mm})$ & 50 & 70 & 90 \\
Normal load $(L),(N)$ & 40 & 60 & 80 \\
RPM of counter disc $(N)$ & 200 & 400 & 600 \\
\hline
\end{tabular}




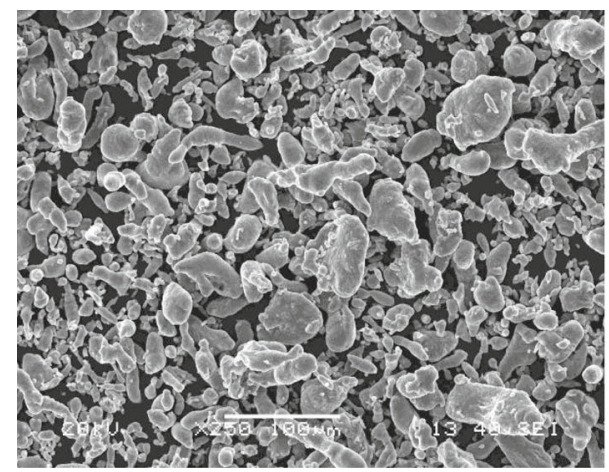

(a)

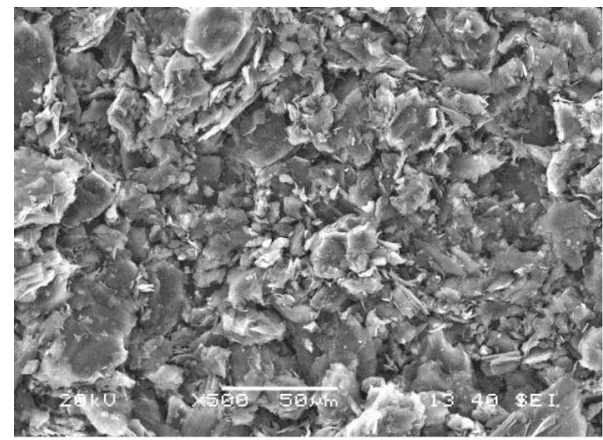

(c)

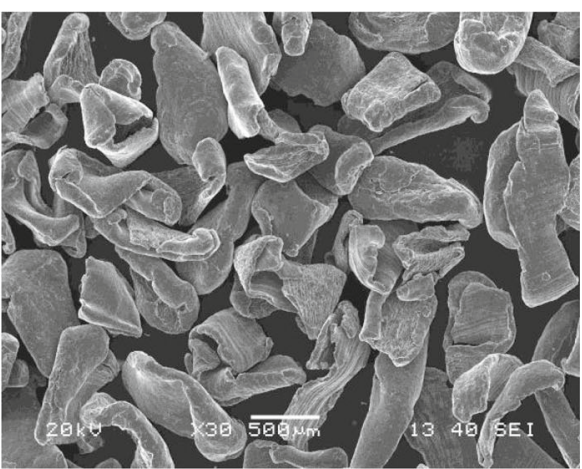

(b)

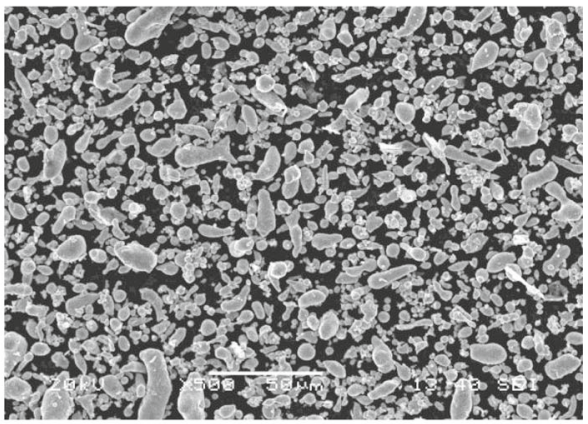

(d)

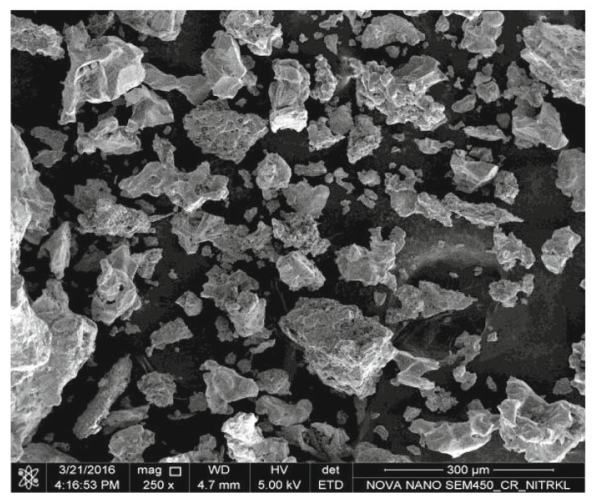

(e)

Fig. 3 SEM image of a aluminium, b magnesium, c graphite, $\mathbf{d}$ zinc and e titanium powder

anterior to experimentation. The variable parameters chosen for the wear analysis are tabulated in Table 2. Ten minutes of test duration was adopted for each experiment. With an accuracy of $0.1 \mathrm{mg}$, the MMC pin (specimen) was weighed before and after the wear operation for determining the wear loss.

\section{Results and discussion}

\section{Physical characteristics}

Flowability of the powder is directly influenced by physical properties as well as environmental conditions of the powders. The mechanical and tribological properties of the final product are directly related to flowability.
Table 3 Physical characteristics of powders

\begin{tabular}{llllc}
\hline Powder & Supplier & $\begin{array}{l}\text { Average size } \\
(\mu \mathrm{m})\end{array}$ & Particle shape & $\begin{array}{l}\text { Purity/assay } \\
(\%)\end{array}$ \\
\hline Aluminium & $\begin{array}{l}\text { Loba } \\
\text { Chemie }\end{array}$ & 45 & $\begin{array}{l}\text { Spherical and } \\
\text { sub-rounded }\end{array}$ & 98.0 \\
Magnesium & 140 & $\begin{array}{l}\text { Flakey } \\
\text { Rounded and } \\
\text { Graphite }\end{array}$ & 20 & $\begin{array}{l}\text { flakey } \\
\text { Spherical and } \\
\text { sub-rounded } \\
\text { Very angular } \\
\text { Zinc }\end{array}$ \\
and irregular & 98.0 \\
Ti & 22 & 98.0 \\
\hline
\end{tabular}




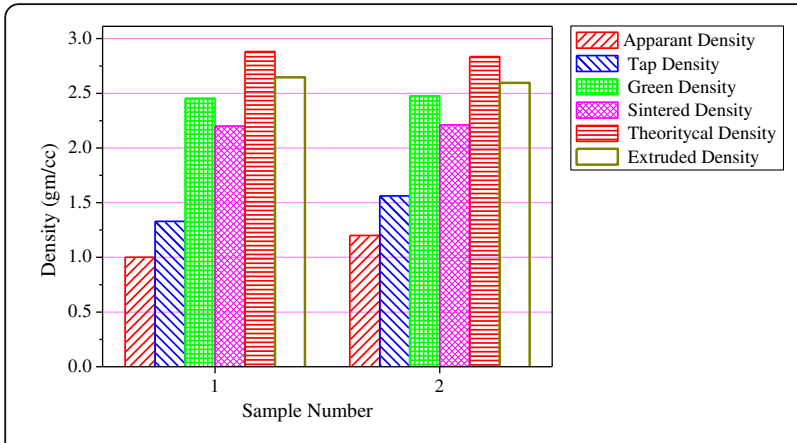

Fig. 4 Density plot

Hence, there is a great importance for the study of physical characterisation such as size, shape and densities of the powders. Among the three most popular techniques used for powder size determination like microscopy, laser diffraction and sieve analysis, microscopy and image analysis were performed for determining the size and shape of the powders. The SEM images of all the powders are shown in Fig. 3.

Image analysis software package is employed for the analysis of particle size and shape of the powders. The detailed report is presented in Table 3.

\section{Density analysis}

Apparent density/bulk density and tap density were recorded from direct measurement. It is depicted from the graph shown in Fig. 4 that $30-35 \%$ improvement of density caused by tapping the blended powder. The green density of the pellet primarily depends on the compaction pressure. It was found a good consolidation of metallic particles even in green specimens at $275 \mathrm{MPa}$. The density was calculated by dividing the measured volume (with the accuracy of $\pm 2 \%$ ) with the measured mass (with the accuracy of $0.001 \mathrm{~g}$ ). The nondimensional densification parameters were calculated by the following relation illustrated in Eq. 3 (Padmavathi et al. 2011).
Table 4 Porosity analysis

\begin{tabular}{lcl}
\hline Sample & Sample 1 & Sample 2 \\
\hline Densification factor & -0.597 & -0.742 \\
$\begin{array}{l}\text { Percentage improvement in densification } \\
\text { (after extrusion) }\end{array}$ & 20.32 & 17.35 \\
Porosity before extrusion (\%) & 23.7 & 22 \\
Porosity after extrusion (\%) & 8.15 & 8.53 \\
\hline
\end{tabular}

Densification parameter $=\frac{(\text { sintered density-green density })}{(\text { theoretical density-green density })}$

Positive densification parameters indicates shrinkage and negative for growth or swelling. All the samples are showing swelling behaviour at the time of sintering. The percentage improvement in densification by thermomechanical treatment (extrusion) is calculated by the following relation illustrated in Eq. 4.

$\begin{aligned} & \text { Percentage improvement } \\ & \text { in densification }\end{aligned}=\frac{\text { extruded density }- \text { sintered density }}{\text { sintered density }}$

There is an increase of $15-20 \%$ of density after extrusion with $50 \%$ reduction evident in Fig. 4. The calculated values of densification factor, improvement of density and porosities are presented in Table 4 . The density of sample type 1 is higher because of higher zinc density.

\section{Compression test of sintered specimen}

Compression test of all the two types of pellets was conducted by UTM (Instron-setec series) with the ram rate of $3 \mathrm{~mm} / \mathrm{min}$ at room temperature. From the output results stress versus strain, curve is plotted in Fig. $5 \mathrm{a}$, b. The average ultimate stress of the two samples is 409 , $381 \mathrm{MPa}$ for samples 1 and 2, respectively. The strength of sample type 1 is on the higher side which can be

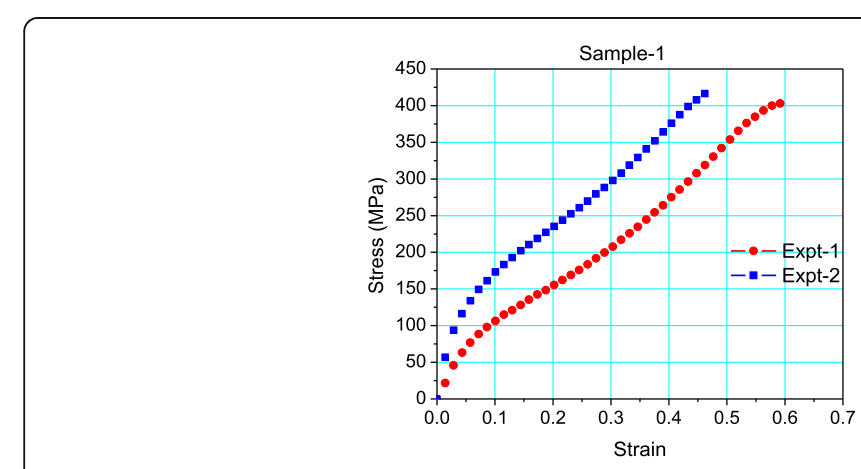

(a)

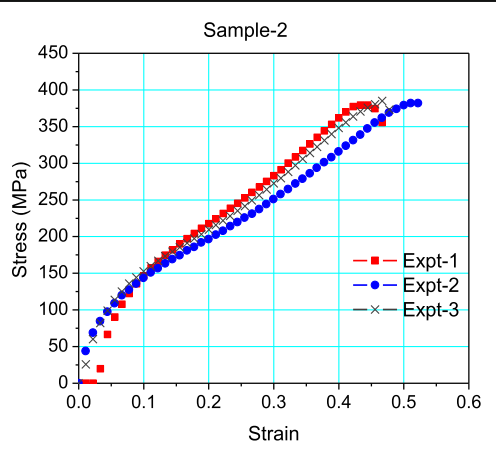

(b)

Fig. 5 Stress versus strain for a sample 1 and $\mathbf{b}$ sample 2 


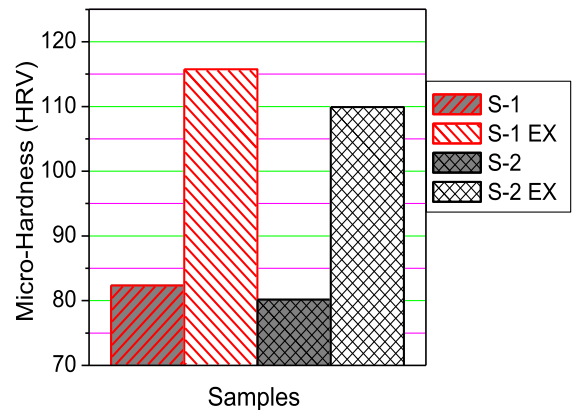

(a)

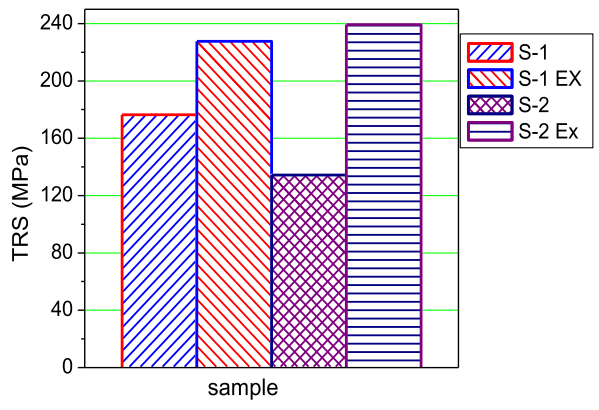

(b)

Fig. $\mathbf{6}$ a Micro-hardness and $\mathbf{b}$ transverse rupture strength

attributed to the excellent bond strength due to liquid phase sintering.

\section{Micro-hardness}

The average micro-hardness was calculated from the ten readings taken for both sintered and extruded products. Figure 6a shows there exists $35-40 \%$ of improvement of hardness in the material after extrusion prepared by the above procedure. It can also be attributed to the higher dislocation density around the reinforcement particles due to the difference of mechanical property and thermal mismatch (Ozdemir and Toparli 2003; Ramesh et al. 2011). The mismatch of the properties between the matrix and reinforcements causes the storage of large internal and thermal stress and engenders improved mechanical properties.

\section{3-point bend test}

The TRS in MPa found of the specimens was estimated for sintered as well as extruded specimen by following the relations mentioned in Eqs. 5 and 6, respectively.

$$
\begin{aligned}
\mathrm{TRS} & =8 P l / \Pi D^{3} \\
\mathrm{TRS} & =3 P l / 2 d^{3}
\end{aligned}
$$

where $P=$ the maximum load $(N)$

$l=$ length of the sample $(\mathrm{mm})$

$D=$ diameter of the sintered specimen $(\mathrm{mm})$

$d=$ depth $=$ width of the extruded square specimen $(\mathrm{mm})$

The average TRS for all sintered and extruded sample is presented in Fig. 6b. For the case of sample type 1,
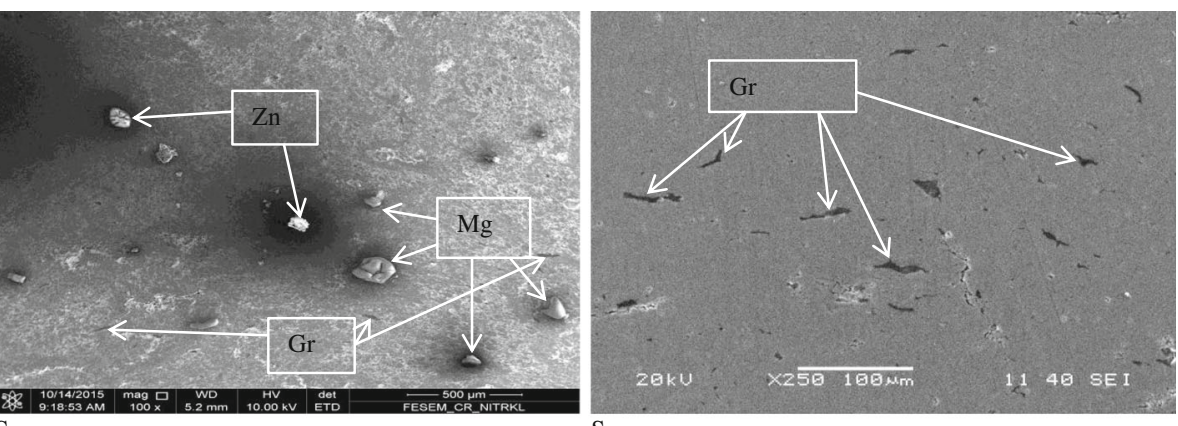

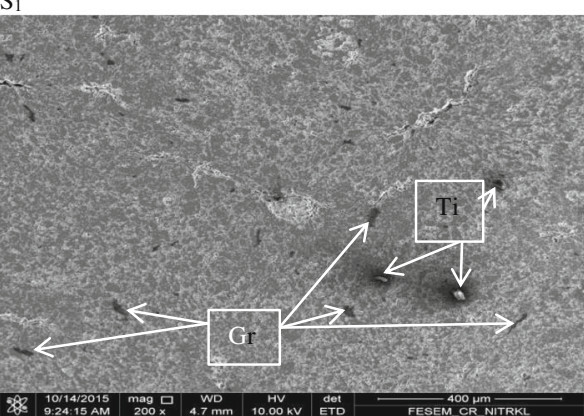

$\mathrm{S}_{2}$

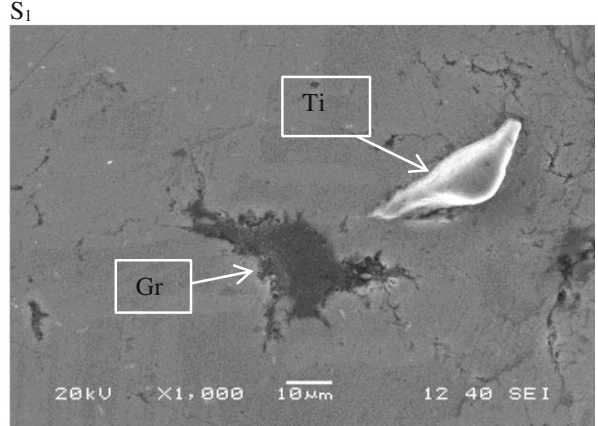

Fig. 7 Microstructural analysis of extruded specimen 
presence of zinc having a melting point of $420^{\circ} \mathrm{C}$ caused liquid phase sintering at a temperature of $600{ }^{\circ} \mathrm{C}$. In the case of Ti-reinforced sample, there exists a high-stress concentration at the boundary zone of the reinforcements and causes the initiation of fracture so comparatively lesser than TRS.

\section{Microstructural analysis}

Figure 7 depicts the scanning electron micrographs of the two types of specimens after the thermo-mechanical treatment at different magnifications. A very little amount of porosity still remains in the material after extrusion which is evident from the figure below. After extrusion operation, the improved density and decreased volume of porosity is illustrated in Fig. 4. The distribution of reinforced particles in the extruded product is uniform and it also shows the good dispersion of reinforcements in matrix elements. There exists a very fine distribution of graphite and other reinforcements like $\mathrm{Mg}, \mathrm{Ti}$ and $\mathrm{Zn}$. There is no observation of rupture of the reinforcing particle (Ti) in sample type 2 due to the compressive pressure exhibited by extrusion. Also, there is a good bond that exists in between $\mathrm{Ti}$ and the matrix element due to its very angular or irregular shape.

\section{Wear test}

Considering the aforementioned three variables with three levels, an orthogonal array (Table 5) has been designed for experimentation of extruded and sintered samples. The variation of wear rates of sintered, as well as extruded specimen for two types of composites, are presented in Figs. 8 and 9. Mass loss of the pin was estimated from the recorded mass of the sample before and after wear test. The mass loss the test is defined as (Soltani et al. 2014):

$$
\Delta w=\left(w_{\mathrm{a}}-w_{\mathrm{b}}\right)
$$

where $w_{\mathrm{a}}$ and $w_{\mathrm{b}}$ are the mass of the sample before and after the test, respectively.

Table $\mathbf{5}$ L9 orthogonal array followed for wear analysis

\begin{tabular}{llll}
\hline Run & Load $(N)$ & Track dia $(\mathrm{mm})$ & RPM of counter disc \\
\hline 1 & 20 & 50 & 200 \\
2 & 20 & 70 & 400 \\
3 & 20 & 90 & 600 \\
4 & 40 & 50 & 400 \\
5 & 40 & 70 & 600 \\
6 & 40 & 90 & 200 \\
7 & 60 & 50 & 600 \\
8 & 60 & 70 & 200 \\
9 & 60 & 90 & 400 \\
\hline
\end{tabular}

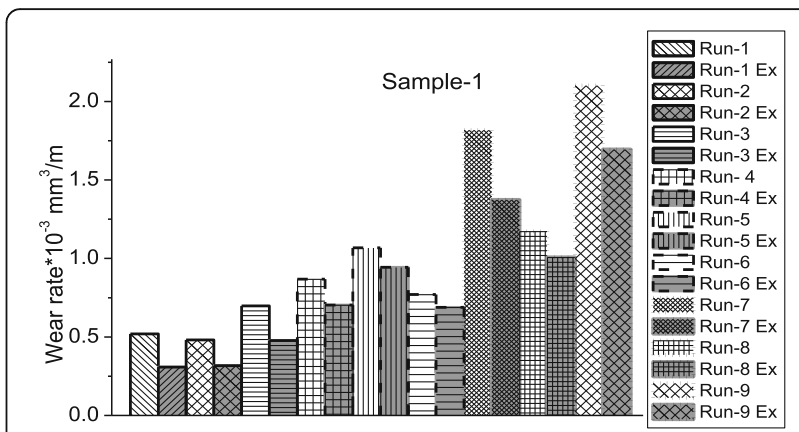

Fig. 8 Wear rate for each run for sample type 1

The volume loss of the AMCs is estimated as the following relation:

Volume loss $\left(\mathrm{mm}^{3}\right)=$ Mass loss $(\mathrm{g}) /$ Density $\left(\mathrm{g} / \mathrm{mm}^{3}\right)$

The volumetric wear rate $\mathrm{W}_{\mathrm{r}}\left(\mathrm{mm}^{3} / \mathrm{Km}\right)$ was estimated by following the relation

$$
\mathrm{W}_{\mathrm{r}}=\text { Volume loss }\left(\mathrm{mm}^{3}\right) / \text { Sliding distance }(\mathrm{Km})
$$

Several researchers have reported the direct proportionality relation of hardness with wear resistance. The bond strength between the matrix and reinforcement material improves after extrusion which improves wear resistance, and it also avoids three-body abrasive wear (Ramesh et al. 1992).

\section{Wear microscopy}

SEM photomicrographs of the AMCs and extruded specimen for run-2 and 7 are shown in Fig. 10. It is observed there is lesser extent of grooving for extruded specimen than the AMCs. The grooves of the unextruded AMCs are coarse and heavy plastic deformation occuring at the groove linings. Also, granular and flakelike debris are observed around the grooves.

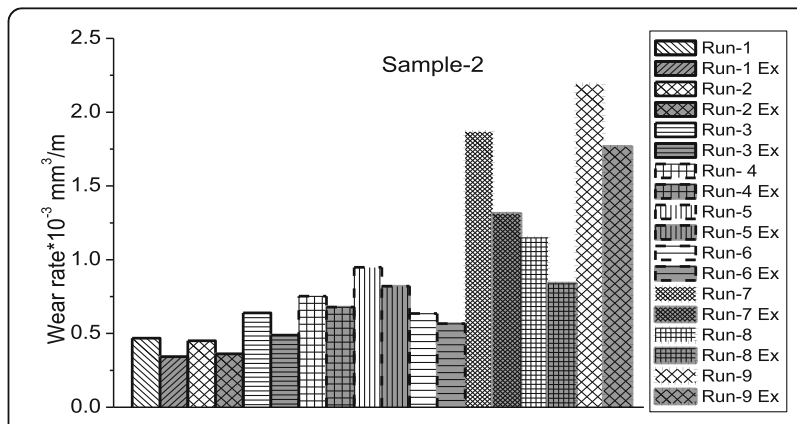

Fig. 9 Wear rate for each run for sample type 2 
At higher loading conditions of 40 and $60 \mathrm{~N}$ with higher RPM of 600 grooving and scratching plays a predominant role over abrasion. The images showing a large amount of white particles present at the tribosurface which can be attributed to the oxidation of the surface due to frictional heating as the aluminium surface is oxide-prone.

At very high loading and high-velocity condition delamination and combination of abrasion, delamination and adhesion mechanism of wear came into the picture.

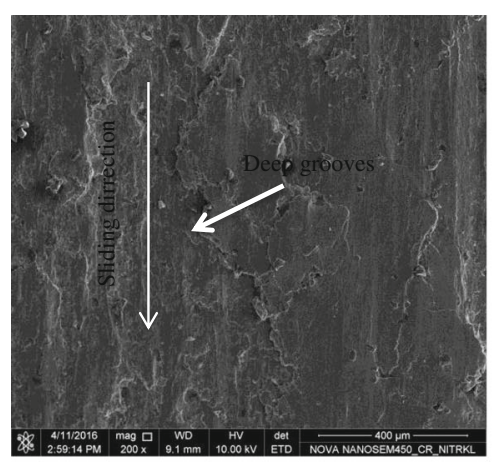

(a) S1 run 2

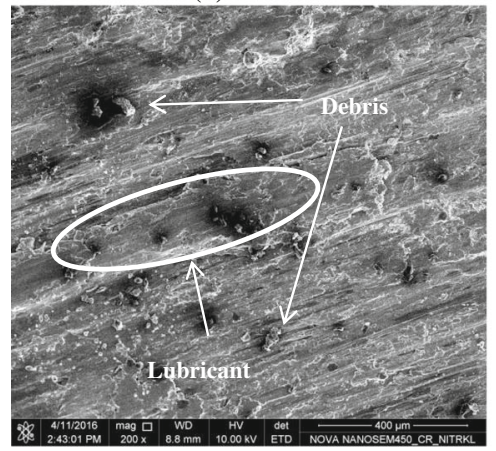

(c) S1 extruded run-2

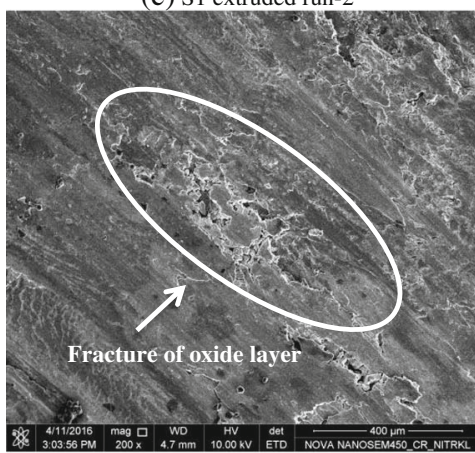

(e) $\mathrm{S} 2$ run-2

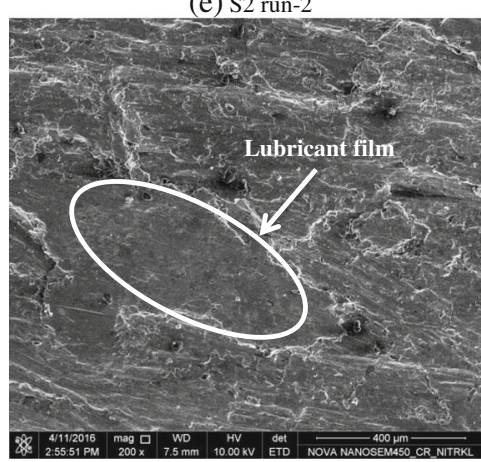

(g) S2 extruded run-2

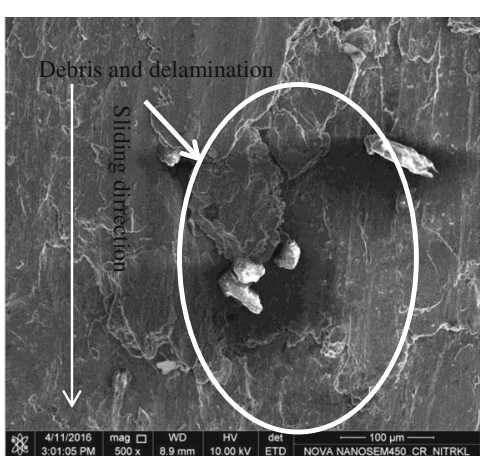

(b) $\mathrm{S} 1$ run7

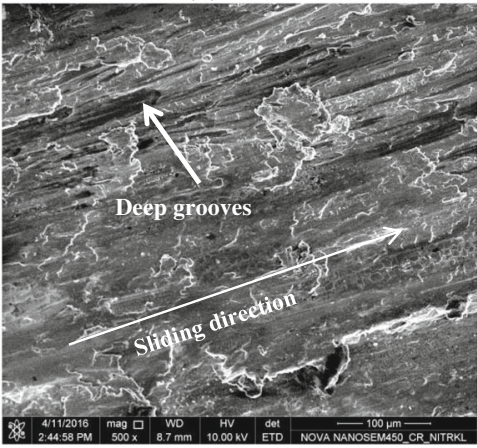

(d) S1 extruded run-7

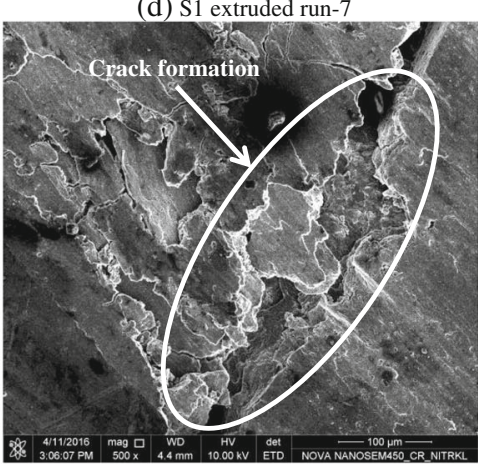

(f) $\mathrm{S} 2$ run-7

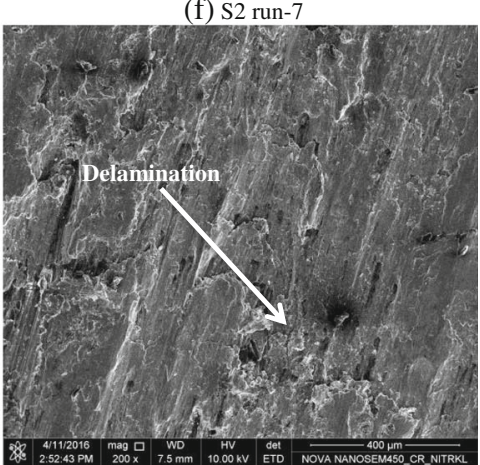

(h) S2 extruded run-7

Fig. 10 SEM micrographs of worn surfaces 
Due to frequent repetitive sliding behaviour, subsurface crack has been induced due to the fatigue failure of the pin. These subsurface cracks grow with increasing travel distance, and eventually, shear deformation occurs to the surface. Moreover, at the adverse conditions, melting, thermal softening and adhesion take the predominant role to cause plastic deformation. In the case of AMCs, the mechanism of wear is less severe than the base metal alloys. Metal/graphite composite forms a lubricating layer on the tribosurface due to the shearing of graphite particles which prevents the metal to metal contact, causing the reduction of friction and wear.

\section{Conclusions}

The effect of extrusion on the improvement of the mechanical and tribological properties of the AMCs was investigated. The results of this investigations are summerised as follows:

(a) Mechanical properties of both types of AMCs are improved due to the improved bond strength after extruding it through mathematically contoured cosine profiled die.

(b) It was found very less amount of surface defects (few cracks at the corner zone) in the extruded product and supports improvement of flexural strength and tribological properties. Wear rate of the extruded specimen is lesser compared to that of the sintered specimen for each run.

(c) The addition of zinc causes liquid phase sintering because of its low melting temperature that leads higher bond strength and density and gives a comparative better performance for the composition. The addition of more amount of fine titanium may improve the properties manifold by compromising with thermal conductivity.

(d) Shearing of the well-distributed graphite particles at the tribosurface acts as a lubricant. So, the addition of graphite particle improves the wear resistance by compromising the little amount of hardness.

(e) At higher loading and sliding velocity condition, a mixed type of wear mechanism (oxidative, delamination, adhesive and abrasion) takes place. But oxidative and delamination are the predominating wear mechanism found on the surface.

\section{Authors' contributions}

All authors read and approved the final manuscript.

\section{Competing interests}

The authors declare that they have no competing interests.

\section{References}

Abdollahi, A., Alizadeh, A., \& Baharvandi, H. R. (2014). Dry sliding tribological behavior and mechanical properties of Al2024-5 wt.\%B4C nanocomposite produced by mechanical milling and hot extrusion. Materials \& Design, 55, 471-481.

Anilkumar, H., Hebbar, H., \& Ravishankar, K. (2011). Mechanical properties of fly ash reinforced aluminium alloy (Al6061) composites. International Journal of Mechanical and Materials Engineering, 6(1), 41-45.

Baradeswaran, A., \& Perumal, A. E. (2014). Wear and mechanical characteristics of Al 7075/graphite composites. Composites Part B: Engineering, 56, 472-476.

El-Kady, O., \& Fathy, A. (2014). Effect of SiC particle size on the physical and mechanical properties of extruded Al matrix nanocomposites. Materials \& Design, 54, 348-353.

Gopalakrishnan, S., \& Murugan, N. (2012). Production and wear characterisation of AA 6061 matrix titanium carbide particulate reinforced composite by enhanced stir casting method. Composites Part B: Engineering, 43(2), 302-308.

Jabbari Taleghani, M. A., Ruiz Navas, E. M., \& Torralba, J. M. (2014). Microstructural and mechanical characterisation of 7075 aluminium alloy consolidated from a premixed powder by cold compaction and hot extrusion. Materials \& Design, 55, 674-682.

Min, K. H., Kang, S. P., Kim, D.-G., \& Kim, Y. D. (2005). Sintering characteristic of Al2O3-reinforced 2xxx series Al composite powders. Journal of Alloys and Compounds, 400(1-2), 150-153.

Ozdemir, I., \& Toparli, M. (2003). An investigation of Al-SiCp composites under thermal cycling. Journal of Composite Materials, 37(20), 1839-1850.

Padmavathi, C., Upadhyaya, A., \& Agrawal, D. (2011). Effect of microwave and conventional heating on sintering behavior and properties of $\mathrm{Al}-\mathrm{Mg}-\mathrm{Si}-\mathrm{Cu}$ alloy. Materials Chemistry and Physics, 130(1), 449-457.

Prasad, V. B., Bhat, B., Mahajan, Y., \& Ramakrishnan, P. (2001). Effect of extrusion parameters on structure and properties of 2124 aluminum alloy matrix composites.

Ramesh, C. S., Seshadri, S. K., \& lyer, K. J. L. (1992). A model for wear rates of composite coatings. Wear, 156(2), 205-209.

Ramesh, C., Keshavamurthy, R., \& Naveen, G. (2011). Effect of extrusion ratio on wear behaviour of hot extruded Al6061-SiC p (Ni-P coated) composites. Wear, 271(9), 1868-1877.

Ravindran, P., Manisekar, K., Narayanasamy, P., Selvakumar, N., \& Narayanasamy, R. (2012). Application of factorial techniques to study the wear of Al hybrid composites with graphite addition. Materials \& Design, 39, 42-54.

Ravindran, P., Manisekar, K., Narayanasamy, R., \& Narayanasamy, P. (2013). Tribological behaviour of powder metallurgy-processed aluminium hybrid composites with the addition of graphite solid lubricant. Ceramics International, 39(2), 1169-1182.

Schatt, W., Association, E.P.M., \& Wieters, KP. (1997). Powder metallurgy: processing and materials. European Powder Metallurgy Association. KIT Scientific Publishing ist Mitglied der Arbeitsgemeinschaft der Universitätsverlage und der Association of European University Presses (AEUP).

Soltani, N., Jafari Nodooshan, H. R., Bahrami, A., Pech-Canul, M. I., Liu, W., \& Wu, G. (2014). Effect of hot extrusion on wear properties of Al-15 wt.\% Mg2Si in situ metal matrix composites. Materials \& Design, 53, 774-781.

Suresha, S., \& Sridhara, B. (2010). Wear characteristics of hybrid aluminium matrix composites reinforced with graphite and silicon carbide particulates. Composites Science and Technology, 70(11), 1652-1659.

Torralba, J. M., da Costa, C. E., \& Velasco, F. (2003). P/M aluminum matrix composites: an overview. Journal of Materials Processing Technology, 133(1-2), 203-206.

Yigezu, B. S., Jha, P., \& Mahapatra, M. (2013). The key attributes of synthesizing ceramic particulate reinforced Al-based matrix composites through stir casting process: a review. Materials and Manufacturing Processes, 28(9), 969-979.

Zhang, C., Zhao, G., Chen, H., Guan, Y., Li, H. (2012a). Optimization of an aluminum profile extrusion process based on Taguchi's method with S/N analysis. The International Journal of Advanced Manufacturing Technology, 60(5), 589-599. doi:10.1007/s00170-011-3622-X

Zhang, C., Zhao, G., Chen, H., Guan, Y., Kou, F. (2012b). Numerical simulation and metal flow analysis of hot extrusion process for a complex hollow aluminum profile. The International Journal of Advanced Manufacturing Technology, 60(1), 101-110. doi:10.1007/s00170-011-3609-7. 